EXTENDED REPORT

\title{
Efficiency of blood culture bottles for the fungal sterility testing of corneal organ culture media
}

\author{
G Thuret, A Carricajo, A C Vautrin, H Raberin, S Acquart, O Garraud, P Gain, G Aubert
}

Br J Ophthalmol 2005;89:586-590. doi: 10.1136/bjo.2004.053439

See end of article for authors' affiliations

Correspondence to: Dr Gilles Thuret, Service d'Ophtalmologie (pavillon 50A), CHRU de Bellevue, 25 Boulevard Pasteur, F 42055 Saint-Etienne Cedex 2, France; gilles. thuret@univ-st-etienne.fr

Accepted for publication 1 September 2004

\begin{abstract}
Background/aim: The consequences of fungal contamination of an organ cultured cornea, though exceptional, are often disastrous for the recipient. Consequently, eye banks often quarantine corneas for 10 days or more before passing them for grafting. This period, though detrimental to the endothelial cell density of the delivered cornea, is necessary to detect contamination using conventional microbiological methods. The authors previously validated the use of a pair of aerobic and anaerobic blood bottles for sensitive and rapid detection of bacteria. To allow a short quarantine period, it remained only to optimise detection of fungi. The authors aimed to compare sensitivity and rapidity of fungal contamination detection by three methods: blood bottles, Sabouraud, and daily visual inspection of the organ culture medium. Methods: Four inocula $\left(10^{6}, 10^{4}, 10^{2}, 10\right.$ colony forming unit (CFU) per ml) of 11 fungi (Candida albicans, C tropicalis, C glabrata, Saccharomyces cerevisiae, Rhodotorula rubra, Cryptococcus neoformans, Fusarium oxysporum, Aspergillus niger, A fumigatus, A flavus, Acremonium falciforme) were inoculated in a commercial organ culture medium containing a coloured pH indicator (CorneaMax, Eurobio, Les Ulis, France). The real live fungal inoculum was verified immediately after inoculation. After 48 hours at $31^{\circ} \mathrm{C}$, samples of the contaminated media were inoculated in three blood bottles: Bactec Aerobic/F, Bactec Mycosis IC/F, and Bactec Myco/F Lytic (Becton Dickinson, Le Pont de Claix, France), then placed in a Bactec 9240 rocking automat, and in four Sabouraud media (solid and liquid, $28^{\circ} \mathrm{C}$ and $37^{\circ} \mathrm{C}$ ) with daily observation. Contaminated organ culture media were also checked daily for any change in turbidity and/or colour. Experiments were performed in triplicate.

Results: Mycosis IC/F and Myco/F Lytic bottles were neither faster nor more sensitive than the aerobic bottle. The three methods were positive for all inocula, even the lowest (viable inoculum below $10 \mathrm{CFU} / \mathrm{ml}$ for each fungus). Contamination was detected within 24 hours by the aerobic bottles in $91 \%$ (40/44), by Sabouraud in $98 \%(43 / 44)$ (no significant difference) and by visual inspection in $66 \%$ of cases $(29 / 44)$ $(p<0.001$ with the two others). Maximum times to detection were 46,48 and 72 hours respectively. Conclusion: This study further counters the preconception that fungal contamination is hard to detect in corneal organ culture media. This study is the last step in validating the use of a pair of blood bottles for the sterility testing of organ culture media, this time for fungi. Their use should make it possible to shorten microbiological quarantine and thus deliver corneas with higher endothelial cell density, without increasing the risk of recipient contamination.
\end{abstract}

rgan culture, as opposed to short term storage at $4^{\circ} \mathrm{C}$ is a cell culture derived method which extends endothelial cell survival of corneal graft tissue and facilitates graft organisation. ${ }^{1}$ However, it creates conditions that promote development of donor micro-organisms that have resisted decontamination of the ocular globe by povidone-iodine. $^{23}$ One or more microbiological tests are thus necessary during storage to ensure that the corneal tissue is delivered uncontaminated for grafting. This safety measure, which is also an advantage of organ culture compared to storage at $4^{\circ} \mathrm{C}^{4-6}$ can take three forms: (i) daily visual inspection of the medium by eye bank technicians searching for a change in transparency, or colour, or the presence of micro-organism colonies; (ii) culturing of a medium sample on broths or conventional agars, observed daily by microbiological laboratory or eye bank technicians; (iii) seeding of a medium sample in blood bottles, which are then placed in an automat performing automatic, continual detection of contamination. We previously showed that the use of a pair of aerobic and anaerobic blood bottles increased the sensitivity and rapidity of detection of bacterial contamination of organ culture media compared to visual inspection and to conventional bacteriological methods.$^{7-9}$ It remained to assess their performance in detecting fungal contamination, in order to definitively validate their use by eye banks, especially as bottles with added sugar and antibiotics designed to improve diagnosis of fungal septicaemia are now available to microbiologists. ${ }^{10}{ }^{11}$ Yeasts and filamentous fungi are of particular concern to ophthalmologists and "eye bankers." Note that fungal endophthalmitis after grafting of an organ cultured cornea partly caused this technique to be quickly discontinued in the United States in 1978. ${ }^{12}$ In addition, fungi are frequently present in postmortem donor flora, and some resist decontamination of the ocular globe by povidone-iodine. ${ }^{213}$ Lastly, certain fungi are reputed to grow slowly on conventional culture media, ${ }^{14}$ which for some eye banks justifies prolonged quarantine before cornea delivery. ${ }^{15}$ According to the 2003 register of the European Eye Bank Association listing the data of the 63 banks (out of 85) using organ culture, 30 banks (48\%) deliver corneas after at least 10 days. ${ }^{16}$ The present study therefore aimed to validate the use of blood bottles to detect fungal contamination of organ culture media.

\section{MATERIALS AND METHODS}

The experiment design, presented in figure 1 , reproduces that used in our previous study on bacterial contamination. ${ }^{8}$

Abbreviations: $\mathrm{CFU}$, colony forming unit 


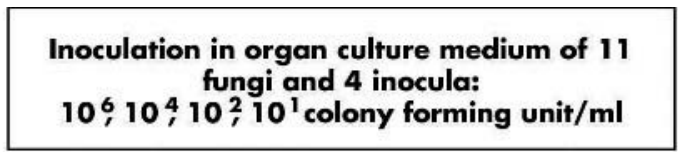

Figure 1 Design of our study. Sabouraud broth and agar were incubated at $28^{\circ} \mathrm{C}$ and $37^{\circ} \mathrm{C}$.
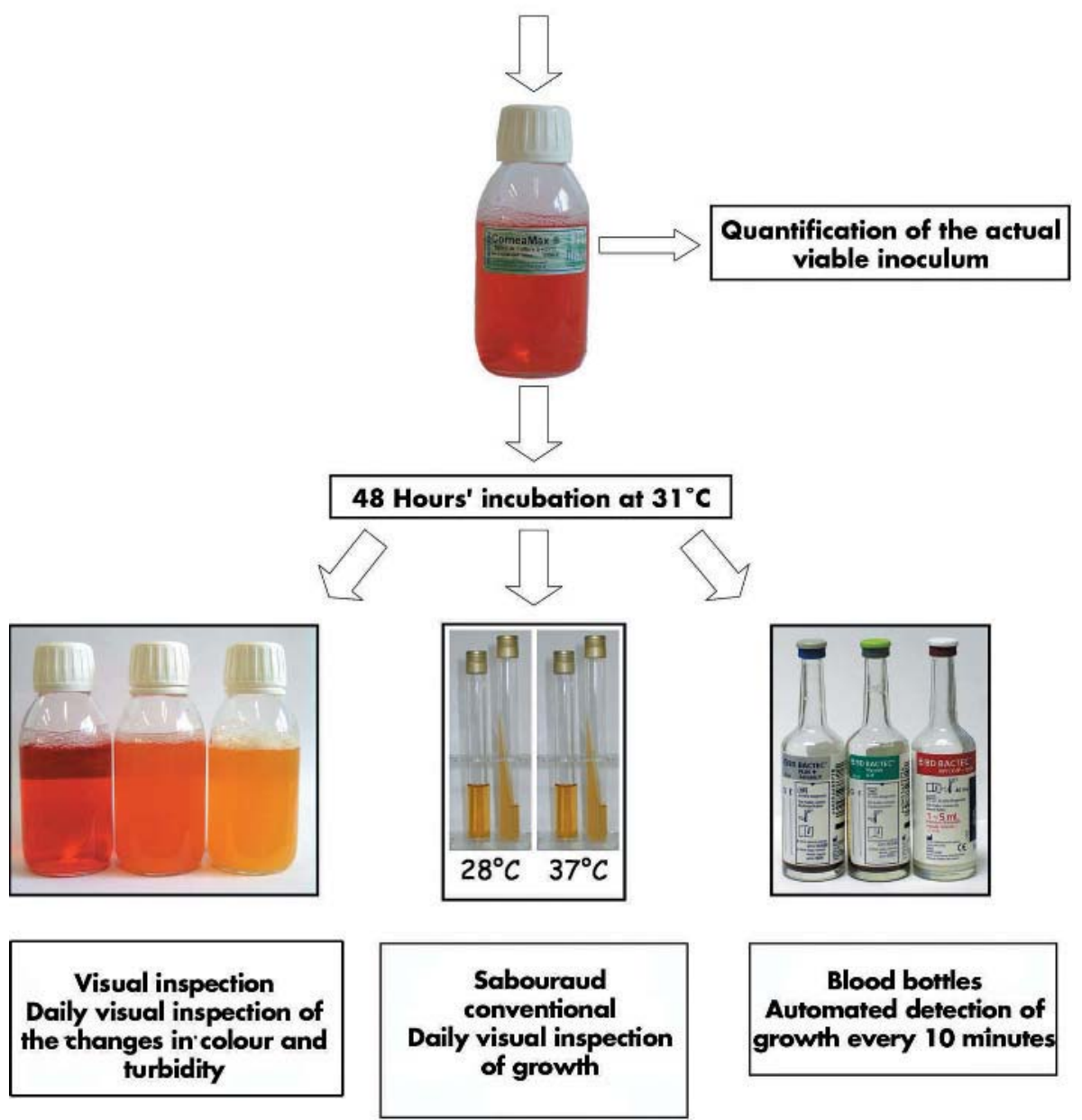

\section{Micro-organisms}

Eleven fungi present in postmortem eye flora ${ }^{13}$ and/or implicated in post-graft endophthalmitis or keratomycosis, generally after storage at $+4^{\circ} \mathrm{C}$ or more rarely in organ culture, were studied. ${ }^{17-25}$ Strains were obtained either from the American Type Culture Collection (ATCC) (Rockville, MD, USA) or the Pasteur Institute (Paris, France): Candida albicans 90028, C tropicalis 66029, C glabrata ATCC 66032, Saccharomyces cerevisiae ATCC 9763, Rhodotorula rubra ATCC 66034, Cryptococcus neoformans 212146, Fusarium oxysporum 625-72, Aspergillus niger 980463435, A fumigatus 864-64, A flavus 97467, Acremonium falciforme 7761.

Four decreasing inocula $\left(10^{6}, 10^{4}, 10^{2}, 10\right.$ colony forming unit $/ \mathrm{ml}(\mathrm{CFU} / \mathrm{ml})$ of each fungus were inoculated in two $100 \mathrm{ml}$ bottles of commercial organ culture medium (CorneaMax, Eurobio, Les Ulis, France). The real inoculum was immediately determined by seeding $100 \mu \mathrm{l}$ of contaminated medium on a Sabouraud medium and counting colonies on the dish. The inoculated organ culture media were incubated in two sealed flasks for 48 hours at $31{ }^{\circ} \mathrm{C}$ in a conventional carbon dioxide free dry incubator. This simulated the initial 2 day quarantine that most European banks routinely observe before the first microbiological tests and sometimes also the first endothelial assessment. The first bottle was then used for culturing on Sabouraud media and

blood bottles, and the second was reserved for the visual method and kept closed.

\section{Microbiological protocols}

In the visual method, changes in colour (to orange or yellow) or turbidity (including the growth of a filamentous fungus in an otherwise clear red medium) of the organ culture medium, indicating positivity, were screened daily by visual inspection until detection. In the Sabouraud method, $1 \mathrm{ml}$ of contaminated organ culture medium was inoculated in two Sabouraud agars and in two Sabouraud broths $(10 \mathrm{ml})$. One Sabouraud set was incubated at $28^{\circ} \mathrm{C}$, the other at $37^{\circ} \mathrm{C}$. Growth was screened daily by visual inspection until positivity. In the blood bottle method, $2.5 \mathrm{ml}$ of contaminated organ culture medium was injected into one Bactec Plus Aerobic/F and two bottles designed for fungal detection: a Bactec Mycosis IC/F and a Bactec Myco/F Lytic (Becton Dickinson, Le Pont de Claix, France). Exceptionally in this series (see below), in the absence of growth of $C$ glabrata in the aerobic bottle, one Bactec Lytic/10 Anaerobic/F bottle was added for this strain. The bottles were placed in a Bactec 9240 incubator at $35^{\circ} \mathrm{C}$ and rocked continuously. The incubator detected any rise in carbon dioxide produced by fungal growth. A sensor placed at the bottom of each bottle reacted with the carbon dioxide and produced fluorescence 
proportional to the carbon dioxide level. Fluorescence was measured every 10 minutes and time to detection was rounded to the nearest hour. All the tests were done in triplicate $(3 \times 11$ fungi $\times 4$ inocula $\times 8$ assays $=1056$ times to detection obtained). For each tested fungus, a negative control was performed for the three detection techniques: a sealed uncontaminated CorneaMax bottle was placed in the incubator at $31^{\circ} \mathrm{C}$ for visual observation, and a second uncontaminated bottle was seeded on Sabouraud and blood bottles. All negative controls were observed for 35 days. In all three methods, identification of isolated micro-organisms was verified from subcultures of the positive media by standard fungal methods. Detection sensitivity and rapidity of the three methods (visual inspection, Sabouraud, blood bottles) were compared.

\section{RESULTS}

The real inocula varied by less than $10-15 \times 10^{6} \mathrm{CFU} / \mathrm{ml}$. The inoculated fungi always corresponded to the one detected, thus ruling out any exogenous contamination during manipulation by the technician.

\section{Performance comparison of the three blood bottles}

The three blood bottles detected all 11 fungi except $C$ glabrata, whose growth was inconsistently detected by the aerobic bottle (only three out of the 12 assays). However, this yeast was always detected by the two fungal bottles and the anaerobic bottle. Times to detection in hours and percentage of detection within the 24 hours following the inoculation, which in practice reflect the working hours of a hospital microbiology laboratory (see paragraph below), are presented in table 1. When comparing these percentages, there was no significant difference between the Aerobic/F and Mycosis IC/F bottles $(p=1)$. The Myco/F Lytic tended to be less effective, but the difference observed did not reach the significance threshold $(p=0.080$ for Aerobic/F and $p=0.156$ for Mycosis IC/F). No blood bottle tested positive without an identifiable germ, thus ruling out the possibility of a false positive having been generated by a physicochemical reaction of the organ culture medium with the contents of the bottle. The results obtained with the aerobic bottle, which tended to be the most effective of the three and in any case routinely used for bacteria, ${ }^{8}$ were then compared with the other two microbiological methods.

\section{Sensitivity and rapidity of the three methods (fig 2)}

The three methods were positive for all inocula of all fungi, even the lowest (numbered systematically $\leqslant 10 \mathrm{CFU} / \mathrm{ml}$ for each of the 11 fungi. No control medium was positive.

To compare the three methods, whose times to detection were not in practice measured in the same way (automatic measurement every 10 minutes for the blood bottles, and screening by a technician every 24 hours for the other two methods) we only considered the positivity rates within 24 hours. This reflects the reality of a microbiology laboratory or eye bank, with detection during working hours from the day after inoculation. Visual inspection detected contamination within 24 hours in only $66 \%$ of cases $(29 / 44)$, whereas Sabouraud (solid or liquid, whatever the temperature) detected it in $98 \%$ (43/44), and the Aerobic/F bottle in $91 \%$ of cases (40/44). Visual inspection was thus significantly slower than the other two methods $(p<0.001)$, whereas the difference between Sabouraud and the Aerobic/F bottle was insignificant $(p=0.360)$. Maximum times to detection for visual inspection, Sabouraud and the Aerobic/F bottle were 72,48 , and 46 hours respectively. With visual inspection, the maximum time to detection was for the lowest inocula of Saccharomyces cerevisiae and Cryptococcus neoformans, detected by Sabouraud and the Aerobic/F bottle in 24 and 46 hours and 24 and 20 hours, respectively. The only case of Sabouraud positive in 48 hours was for the lowest inoculum of Acremonium falciforme, which was detected by the Aerobic/F bottle in 20 hours. The maximum time to detection by the Aerobic/F bottle (46 hours) was for the lowest inoculum of Saccharomyces cerevisiae, detected in 24 hours by Sabouraud.

\section{DISCUSSION}

This experimental study of the main fungi in postmortem eye flora and/or responsible for several ocular infection complements that done on bacteria. ${ }^{8}$ It validates the use of a pair of aero/anaerobic blood bottles to ensure the microbiological safety of organ culture stored corneas. The three test bottles detected the very low fungal inocula $(\leqslant 10 \mathrm{CFU} / \mathrm{ml})$, which probably corresponds to those found clinically in donors and remaining after decontamination by povidone-iodine. Our methodology, with preparation of inoculum by dilution and immediate verification of the real live inoculum, allowed confirmation that very low starting inoculum had been cultured. These inocula were even lower than those described by Rousset et al, who studied the effectiveness of the visual and Sabouraud methods. ${ }^{26}$ As expected, the aerobic bottle proved very effective on its own for all strains except Candida glabrata. This yeast is difficult for aerobic bottles to detect, as described by studies of series of experimental septicaemias ${ }^{27}$ and actual ones. ${ }^{28}$ However, the yeast was detected by the other blood bottles, particularly the anaerobic one, which is routinely used for bacterial detection in a growing number of French and European eye banks. However, the conventional (Sabouraud, whichever type) and visual methods never appeared inadequate for fungal detection, contrary to our previous study on bacteria using a similar design. ${ }^{8}$ That study showed that the main advantage of the aero/anaerobic blood bottle pair over conventional microbiological techniques or visual inspection is its rapid detection of contamination. This also applies to fungi, because detection by aerobic bottle was obtained in a mean 13 hours (maximum 46). However, this short time was a less decisive advantage than with bacteria. If we reason in laboratory technicians' working hours, time to detection by the other two methods is comparable because detection can always be obtained 1 or 2 days after inoculation (weekends excepted). It was thus necessary to demonstrate that the benefit in terms of rapidity of bacterial detection was not achieved to the detriment of its effectiveness to detect fungi. The present study proves that the pair of blood bottles must be effective enough to allow risk free discontinuation of the Sabouraud medium, which moreover is time consuming for technicians.

Table 1 Comparison of the three blood bottles. Both times to detection in hours and percentage of detection within the 24 hours following inoculation were presented. Results were means of experiments performed in triplicates

\begin{tabular}{llll}
\hline & +Aerobic/F & Mycosis IC/F & Myco/F Lytic \\
\hline $\begin{array}{l}\text { Time to detection (mean (SD), median, range) } \\
\text { \% of detection within 24 hours (n) }\end{array}$ & $13(9), 13,1-46$ & $14(19), 11,1-108$ & $22(24), 14(5-114)$ \\
\hline
\end{tabular}




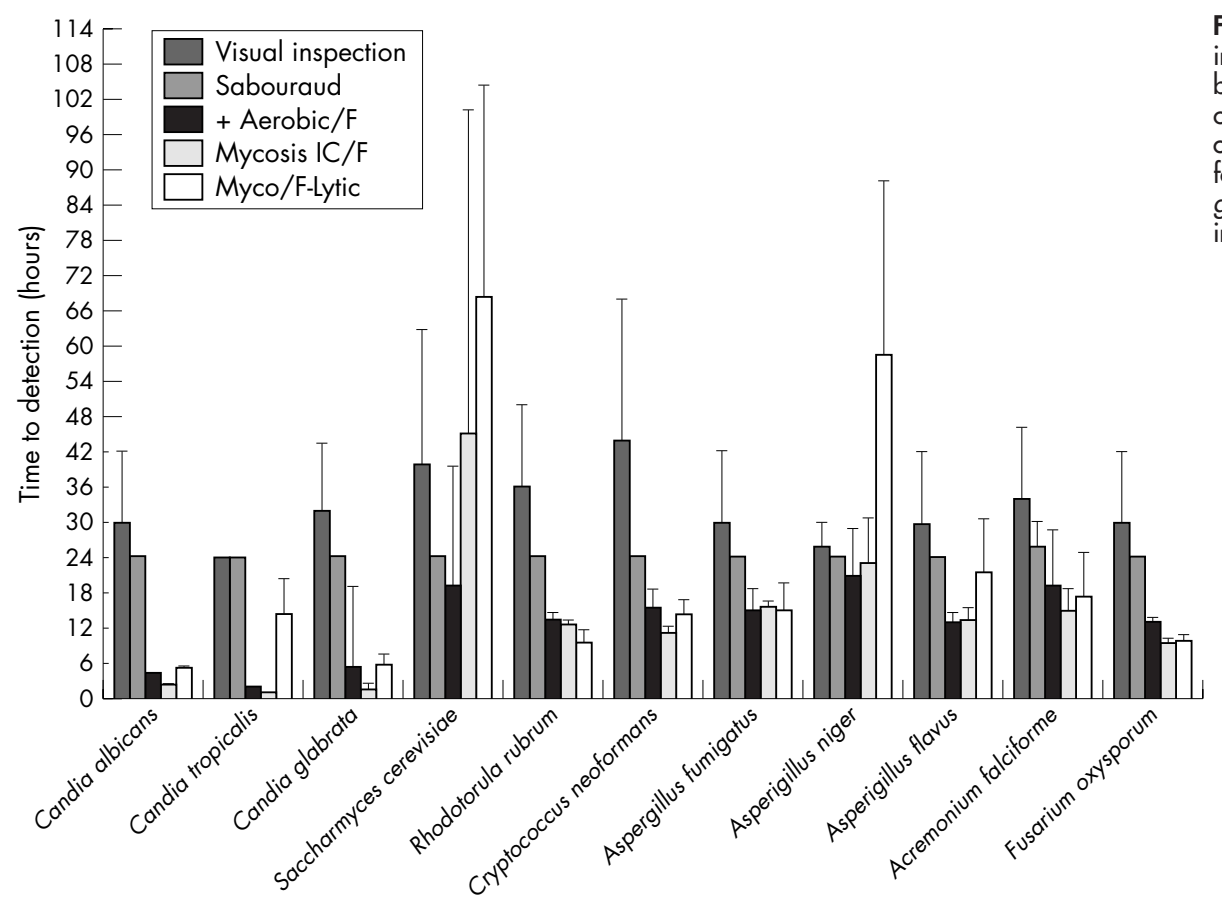

Figure 2 Time to detection by visual inspection, Sabouraud and the three blood bottles. For each method,

columns represent the mean (bars $=S D$ ) of the set of times to detection of the four decreasing inocula. For Candida glabrata. Anaerobic F/Lytic was used instead of +Aerobic/F.

Importantly, all three techniques allowed detection of the lowest inoculum of all the tested fungi within 3 days. Contaminated corneas could thus be very quickly removed from the incubator, minimising the risk of contamination of the bank environment. The high capacity of conventional methods for detecting fungal contamination was stressed by Rousset et al. ${ }^{26}$ Note that neither Rousset's study (on, notably, four fungi not tested in our study: Candida parapsilosis, Absidia spp, Penicillium spp. Trichoderma) nor our own (which tested Saccharomyces cerevisiae, Rhodotorula rubra, Aspergillus niger, not studied by Rousset) did not highlight difficulties in rapid detection of fungal contamination. Inocula were very low in both studies: 10 elements per bottle for Rousset and less than $10 \mathrm{CFU} / \mathrm{ml}$ of viable inoculum in our study. These studies invalidated older ones, ${ }^{14}{ }^{15}$ which raised the fear of late development of pathogenic fungi and so justified prolonged quarantine of graft tissue. Shorter quarantine is currently an important objective, at least in certain penetrating keratoplasty indications. It has been clearly shown ${ }^{29-31}$ that, to increase graft tissue survival in recipients, it is preferable to deliver corneas with the highest possible endothelial cell density. ${ }^{32}{ }^{33}$ This particularly applies to recipients with high endothelial cell density and a low risk of rejection, such as those with keratoconus, ${ }^{31}$ currently one of the main indications for cornea graft in Europe. ${ }^{34}$

Lastly, although a pair of aero/anaerobic bottles costs more than conventional media, the reduction in technician time allowed by task automation is ultimately cost effective and may be a considerable advantage, particularly for banks that analyse large quantities of storage liquids.

\section{CONCLUSION}

The use of a pair of Bactec aerobic and anaerobic blood bottles placed in an automat has enabled us to eliminate the use of Sabouraud medium and, more than 1 year ago, to reduce quarantine by 5 days. ${ }^{16}$ Our patients have thus received corneas with higher endothelial cell density without compromising their bacterial or fungal safety. To remain vigilant, particularly in the hypothesis of exceptional contamination by fungi other than those tested in the present study, we still use a Mycosis/IF bottle, the most effective after the aerobic bottle in our study. We will continue this practice until an observational study of the long term cost/benefit ratio, as for bacteria, ${ }^{9}$ proves whether it should be discontinued.

\section{ACKNOWLEDGEMENTS}

We thank the "Direction Regionale de la Recherche Clinique ( $\mathrm{Pr} \mathrm{H}$ Decousus)" for funding. Grant: Bellevue Hospital 2002 local invitation to tender.

\section{Authors' affiliations}

G Thuret, P Gain, Department of Ophthalmology, Bellevue Hospital, 25 Bd Pasteur, F 42055 Saint-Etienne Cedex 2, France

G Thuret, P Gain, Cell Adherence And Survival In Cancers And Grafts Laboratory, EA3063, Faculty of Medicine, F 42055 Saint-Etienne Cedex 2, France

A Carricajo, A C Vautrin, G Aubert, Department of Microbiology, Bellevue Hospital, 25 Bd Pasteur, F 42055 Saint-Etienne Cedex 2, France

H Raberin, Department of Parasitology, Bellevue Hospital, 25 Bd Pasteur, F 42055 Saint-Etienne Cedex 2, France

S Acquart, O Garraud, French Blood Center, Eye Bank of Saint Etienne, 25 Bd Pasteur, F 42055 Saint-Etienne Cedex 2, France

Proprietary interest: the authors do not have any proprietary interest in the materials used in this study.

Part of this paper was presented at the 16th annual meeting of the European Eye Bank Association, held on 15-17 January 2004 in Barcelona, Spain.

\section{REFERENCES}

1 Doughman DJ, Harris JE, Schmitt MK. Penetrating keratoplasty using $37^{\circ} \mathrm{C}$ organ cultured cornea. Trans Am Acad Ophthalmol Otolaryngol 1976;81:778-93.

2 Pels E, Vrensen GF. Microbial decontamination of human donor eyes with povidone-iodine: penetration, toxicity, and effectiveness. Br J Ophthalmol 1999:83:1019-26.

3 Mindrup EA, Dubbel PA, Doughman DJ. Betadine decontamination of donor globes. Cornea 1993; 12:324-9.

4 Pels L. Organ culture: the method of choice for preservation of human donor corneas. Br J Ophthalmol 1997:81:523-5.

5 Borderie VM, Scheer S, Touzeau O, et al. Donor organ cultured corneal tissue selection before penetrating keratoplasty. $\mathrm{Br} J$ Ophthalmol 1998;82:382-8. 
6 Borderie VM, Laroche L. Microbiologic study of organ-cultured donor corneas. Transplantation 1998;66:120-3.

7 Gain P, Thuret G, Chiquet C, et al. Use of a pair of blood culture bottles for sterility testing of corneal organ culture media. $\mathrm{Br} J$ Ophthalmol 2001:85:1158-62.

8 Thuret G, Carricajo A, Chiquet C, et al. Sensitivity and rapidity of blood culture bottles in the detection of cornea organ culture media contamination by bacteria and fungi. Br J Ophthalmol 2002;86:1422-7.

9 Thuret G, Carricajo A, Chiquet $C$, et al. Optimizing microbiological controls of corneal organ culture media. J Fr Ophtalmol 2003;26:792-800.

10 Vetter E, Torgerson C, Feuker A, et al. Comparison of the Bactec Myco/F Lytic bottle to the isolator tube, Bactec Plus Aerobic F/bottle, and Bactec Anaerobic Lytic/10 bottle and comparison of the Bactec Plus Aerobic F/bottle to the Isolator tube for recovery of bacteria, mycobacteria, and fungi from blood. $J$ Clin Microbiol 2001;39:4380-6.

11 Vigano EF, Vasconi E, Agrappi C, et al. Use of simulated blood cultures for time to detection comparison between BacT/Alert and Bactec 9240 blood culture systems. Diagn Microbiol Infect Dis 2002;44:235-40.

12 Larsen PA, Lindstrom RL, Doughman DJ. Torulopsis glabrata endophthalmitis after keratoplasty with an organ-cultured cornea. Arch Ophthalmol 1978;96:1019-22.

13 Dixon DM, Graham CR Jr, Shaffer RM, et al. Fungal flora from diabetic and non-diabetic human donor corneas. Cornea 1984;3:281-4.

14 Jendral G, Wilhelm $\mathrm{F}$, Bernhardt $\mathrm{H}$, et al. Difficulties of fungus detection in corneal culture medium. Ophthalmologe 1999:96:465-7.

15 Nelson JD, Mindrup EA, Chung CK, et al. Fungal contamination in organ culture. Arch Ophthalmol 1983;101:280-3.

16 EEBA. European Eye Bank Association Directory, 12th ed., 2004.

17 Ando N, Takatori K. Keratomycosis due to Alternaria alternata corneal transplant infection. Mycopathologia 1987;100:17-22.

18 Behrens-Baumann W, Ruechel R, Zimmermann O, et al. Candida tropicalis endophthalmitis following penetrating keratoplasty. $\mathrm{Br} J$ Ophthalmol 1991:75:565.

19 Antonios SR, Cameron JA, Badr IA, et al. Contamination of donor cornea: postpenetrating keratoplasty endophthalmitis. Cornea 1991;10:217-20.
20 Kennedy SM, Shankland GS, Lee WR, et al. Keratitis due to the fungus Acremonium (Cephalosporium). Eye 1994;8:692-4.

21 Sridhar MS, Garg P, Bansal AK, et al. Aspergillus flavus keratitis after laser in situ keratomileusis. Am J Ophthalmol 2000;129:802-4

22 Tixier J, Bourcier T, Borderie V, et al. Infectious keratitis after penetrating keratoplasty. J Fr Ophtalmol 2001;24:597-602.

23 Sridhar MS, Gopinathan U, Garg P, et al. Aspergillus fumigatus keratitis with wreath pattern infiltrates. Cornea $2001 ; 20: 534-5$.

24 Narang S, Gupta A, Gupta V, et al. Fungal endophthalmitis following cataract surgery: clinical presentation, microbiological spectrum, and outcome. Am J Ophthalmol 2001;132:609-17.

25 Sutphin JE, Pfaller MA, Hollis RJ, et al. Donor-to-host transmission of Candida albicans after corneal transplantation. Am J Ophthalmol 2002;134:120-1.

26 Rousset A, Piarroux R, Reboux $G$, et al. Fungal contamination detection in cornea preservation media. J Fr Ophtalmol 2002;25:590-3.

27 Horvath LL, Hospenthal DR, Murray CK, et al. Detection of simulated candidemia by the Bactec 9240 system with plus aerobic/F and anaerobic/F blood culture bottles. J Clin Microbiol 2003;41:4714-17.

28 Meyer MH, Letscher-Bru V, Jaulhac B, et al. Comparison of Mycosis IC/F and plus Aerobic/F media for diagnosis of fungemia by the bactec 9240 system. J Clin Microbiol 2004;42:773-7.

29 Nishimura JK, Hodge DO, Bourne WM. Initial endothelial cell density and chronic endothelial cell loss rate in corneal transplants with late endothelia failure. Ophthalmology 1999; 106:1962-5.

30 Bourne WM. Cellular changes in transplanted human corneas. Cornea $2001 ; 20: 560-9$.

31 Thuret G, Chiquet C, Bernal F, et al. Prospective, randomized clinical and endothelial evaluation of 2 storage times for cornea donor tissue in organ culture at 31 degrees C. Arch Ophthalmol 2003;121:442-50.

32 Thompson RW Jr, Price MO, Bowers PJ, et al. Long-term graft survival after penetrating keratoplasty. Ophthalmology 2003;110:1396-402.

33 Armitage WJ, Dick AD, Bourne WM. Predicting endothelial cell loss and longterm corneal graft survival. Invest Ophthalmol Vis Sci 2003:44:3326-31.

34 Poinard C, Tuppin P, Loty B, et al. The French national waiting list for keratoplasty created in 1999: patient registration, indications, characteristics, and turnover. J Fr Ophtalmol 2003;26:911-19. 